\title{
Strongly localized moving discrete dissipative breather-solitons in Kerr nonlinear media supported by intrinsic gain
}

\author{
Magnus Johansson, Jaroslaw E. Prilepsky and Stanislav A. Derevyanko
}

\section{Linköping University Post Print}

\section{Tweet}

N.B.: When citing this work, cite the original article.

Original Publication:

Magnus Johansson, Jaroslaw E. Prilepsky and Stanislav A. Derevyanko, Strongly localized moving discrete dissipative breather-solitons in Kerr nonlinear media supported by intrinsic gain, 2014, Physical Review E. Statistical, Nonlinear, and Soft Matter Physics, (89), 4, 042912.

http://dx.doi.org/10.1103/PhysRevE.89.042912

Copyright: American Physical Society

http://www.aps.org/

Postprint available at: Linköping University Electronic Press

http://urn.kb.se/resolve?urn=urn:nbn:se:liu:diva-106961 


\title{
Strongly localized moving discrete dissipative breather-solitons in Kerr nonlinear media supported by intrinsic gain
}

\author{
Magnus Johansson, ${ }^{1, *}$ Jaroslaw E. Prilepsky, ${ }^{2}$ and Stanislav A. Derevyanko ${ }^{3}$ \\ ${ }^{1}$ Department of Physics, Chemistry and Biology (IFM), Linköping University, SE-581 83 Linköping, Sweden \\ ${ }^{2}$ Aston Institute of Photonic Technologies, Aston University, B4 7ET, Birmingham, UK \\ ${ }^{3}$ Department of Physics of Complex Systems, Weizmann Institute of Science, Rehovot 76100, Israel
}

(Received 30 December 2013; published 18 April 2014)

\begin{abstract}
We investigate the mobility of nonlinear localized modes in a generalized discrete Ginzburg-Landau-type model, describing a one-dimensional waveguide array in an active Kerr medium with intrinsic, saturable gain and damping. It is shown that exponentially localized, traveling discrete dissipative breather-solitons may exist as stable attractors supported only by intrinsic properties of the medium, i.e., in the absence of any external field or symmetry-breaking perturbations. Through an interplay by the gain and damping effects, the moving soliton may overcome the Peierls-Nabarro barrier, present in the corresponding conservative system, by self-induced time-periodic oscillations of its power (norm) and energy (Hamiltonian), yielding exponential decays to zero with different rates in the forward and backward directions. In certain parameter windows, bistability appears between fast modes with small oscillations and slower, large-oscillation modes. The velocities and the oscillation periods are typically related by lattice commensurability and exhibit period-doubling bifurcations to chaotically "walking" modes under parameter variations. If the model is augmented by intersite Kerr nonlinearity, thereby reducing the Peierls-Nabarro barrier of the conservative system, the existence regime for moving solitons increases considerably, and a richer scenario appears including Hopf bifurcations to incommensurately moving solutions and phase-locking intervals. Stable moving breathers also survive in the presence of weak disorder.
\end{abstract}

DOI: 10.1103/PhysRevE.89.042912

PACS number(s): 05.45.-a, 42.65.Wi, 63.20.Pw, 63.20.Ry

\section{INTRODUCTION}

The concept of dissipative solitons, being localized, dynamical objects with nontrivial internal energy flows which may exist in open, nonlinear systems due to a balance among gain, loss, dispersion or diffraction, and nonlinearity, is by now established in many branches of physics [1,2], and in particular in optics where many applications have been devised [3]. Although originally conceived for spatially continuous systems, they have lattice counterparts as discrete dissipative solitons (or breathers), which also have been discussed in a large number of physical contexts (see, e.g., the review [4]).

In order to provide a feasible mechanism for transport of localized quantities of energy, mobile dissipative solitons are highly important objects. While their existence as exponentially localized modes for continuous systems is well known and not surprising (see, e.g., [5]), the issue is more delicate for lattices where, for the corresponding conservative systems, the lattice can be viewed as inducing a periodic Peierls-Nabarro (PN) potential, which must be overcome during the motion. As a consequence, in a generic conservative lattice system moving localized modes are not expected to exist as exact solutions, since their motion in the PN potential causes radiation to be emitted and thereby decay of a single moving soliton (breather) (see, e.g., Ref. [6] for discussion and further references and Ref. [7] for a mathematical treatment). However, in a dissipative environment with energy input, one might hope that a balance (at least in average) between gain and loss could still be established to sustain a moving discrete soliton

\footnotetext{
*mjn@ifm.liu.se; https://people.ifm.liu.se/majoh
}

indefinitely, and at the same time damp out its radiation into a tail exponentially decaying to zero.

In fact, scenarios similar to that outlined above have already been observed in several contexts, theoretically and numerically as well as experimentally. Probably the first thorough numerical studies of mobile discrete breathers in dissipative lattices were reported in a series of papers by the Zaragoza group [8-11], for the damped and ac-driven discrete sine-Gordon (Frenkel-Kontorova) model. They were found to appear as dynamical attractors for a rather large range of parameters, with phonon tails exponentially decaying due to the damping and asymmetric due to Doppler shifts of radiation emitted in forward and backward directions. Two different types of moving breathers, with a small regime of bistability, were discussed in Refs. [8-11]: "induced fast" breathers, generated from superthreshold, symmetry-breaking dynamical perturbations of stable, pinned breathers, and "spontaneous slow" breathers appearing from depinning parametric instabilities of pinned, quasiperiodic breathers. An intermediate regime with breathers moving in a seemingly random, diffusive way was also identified. Other properties of moving breathers described were the possibility for formation of bound states, and regimes of mode locking of the breather velocity at rational multiples of the driving frequency. However, one should note that due to the spatially homogeneous driving force, the oscillation amplitude of the breather tails does not decay to zero but to some constant value, and thus for an infinite system all those excitations would have an infinite energy.

In optics, discrete dissipative solitons are probably most commonly discussed for arrays of coupled-waveguide resonators, "discrete cavity solitons" (DCSs) [12], for which the standard coupled-mode equations, in the case of Kerr nonlinearity, share many basic features with the small-oscillation 
limit of the damped-driven Frenkel-Kontorova model. In Ref. [13] it was found that resting DCSs could be made mobile by imposing a symmetry-breaking perturbation on the system by inclining the holding beam, thereby introducing a phase gradient for the effective driving force. Further numerical analysis for a similar system with saturable nonlinearity [14] also reached the conclusion that, generally, sufficiently large symmetry breaking was needed for a stable stationary soliton to move. This scenario should be analogous to that of the "induced fast" breathers of Refs. [8-11], except that the symmetry-breaking perturbation is applied to the physical system rather than to the initial condition. A more recent work [15] also identifies "spontaneously walking," as well as uniformly moving, DCSs in the absence of external symmetry breaking, in regimes of Hopf instability of stationary DCSs. These are thus analogous to the diffusively moving and "spontaneous slow" breathers, respectively, of Refs. [8-11].

Experimentally, moving discrete dissipative breathers have been observed in damped-driven electrical lattices, both one dimensional (1D) [16,17] as well as more recently two dimensional (2D) [18]. In the 1D lattice in Ref. [16], traveling breathers locked to a uniform driver were found as generic nonlinear excitations in certain parameter regimes, with velocities precisely tunable by the driver amplitude and frequency. The mechanism for mobility was explained in Ref. [17] in terms of a self-induced dc distortion "propelling" the breather. In 2D [18], an erratic motion was found, comparable to that from [8,9] discussed above.

However, in all the cases discussed above, the moving discrete dissipative solitons (breathers) were supported by uniform external driving, leading necessarily to modes with nonzero tails and infinite energy. By contrast, in this work we address the question of whether moving nonlinear localized lattice modes may be supported solely by an intrinsic gain mechanism, thereby allowing for finite-energy solutions with tails decaying to zero. Although the question is of generic nature, we address it here in the context of a generalized discrete Ginzburg-Landau-type model, introduced by Rozanov's group $[19,20]$ to describe the propagation of monochromatic radiation in a system of weakly coupled single-mode active optical fibers, characterized by saturable amplification and absorption [21] and a Kerr nonlinear refractive index. The properties of stationary, strongly localized discrete solitons in this system were studied in Ref. [20], generalizing earlier results obtained in a cubic-quintic approximation [22,23]. Indeed, also moving solitons were reported for this system in Ref. [19], but only for a special case with zero Kerr nonlinearity, and with broad, continuum-like shapes. By contrast, here we mainly focus on showing that also strongly localized moving solitons exist in presence of a standard, onsite Kerr nonlinearity, and illustrate the mechanisms by which the interplay by gain and damping makes it possible to overcome the Peierls-Nabarro barrier of the corresponding conservative system, through self-induced time-periodic oscillations. It should be noted that similar self-induced oscillations of a moving soliton were recently also noticed [24] in the transient dynamics for a continuous model with linear gain and nonlinear loss and a spatially periodic modulation of the linear as well as the Kerr nonlinear refractive index; however, due to the linear gain the background of these solutions were unstable and therefore they were not dynamical attractors.

In Sec. II we describe the generalized discrete GinzburgLandau model of Refs. [19,20], which we also extend with possible intersite Kerr nonlinearities and onsite disorder. We show and discuss our main numerical results in Sec. III. Moving solitons for the standard onsite Kerr nonlinearity are described in Sec. III A, while additional features appearing in the presence of intersite nonlinearities are discussed in Sec. III B, and the survival of the moving solitons also in the presence of weak disorder is shown in Sec. III C. Finally, some concluding remarks are made in Sec. IV.

\section{MODEL}

The model to be used in this paper is a generalization of the model from Ref. [20], which we take in the following form:

$$
\begin{aligned}
& i \dot{\psi}_{n}+C\left(\psi_{n-1}+\psi_{n+1}\right)+\left[V_{n}+\left|\psi_{n}\right|^{2}-i f_{d}\left(\left|\psi_{n}\right|^{2}\right)\right] \psi_{n} \\
& \quad+Q f_{i s}\left(\left\{\psi_{n}\right\}\right)=0 .
\end{aligned}
$$

Here, the dot will be referred to as a time derivative, although evidently it should be interpreted as a derivative with respect to a longitudinal coordinate $z$ for the physical system of coupled identical fibers in Ref. [20], describing discrete spatial optical solitons. In this model, $\psi_{n}$ is the mode amplitude in the $n$th fiber, and $C$ is the coupling constant between neighboring fibers, which we here take to be real and thus neglect losses in the medium between the fibers (in Refs. [19,20], possible absorption or gain in this medium was also considered by adding a small imaginary part to $C$ ). We have also included a linear, real onsite potential $V_{n}$ to allow for possible disorder in the fiber properties (although we take $V_{n} \equiv 0$ for most of the paper). The real function $f_{d}(x)$ describes the amplification and absorption characteristics of each fiber, and is taken as in Refs. [19-21] to have the form

$$
f_{d}(x)=-\delta+\frac{g}{1+x}-\frac{a}{1+b x}, \quad \delta, g, a, b>0 .
$$

Here, $\delta$ describes the linear, nonresonant losses, $g$ and $a$ are the strengths of the saturable gain and absorption, respectively, and $b$ is the ratio between the gain and absorption saturation intensities. We have chosen to normalize the field according to the gain saturation intensity and without loss of generality assumed that the Kerr coefficient is equal to unity. A related model with purely linear losses $(a=0)$ was also studied in Ref. [25] in the context of DCSs (i.e., with an additional constant term in the right-hand side describing the amplitude of a holding beam), where also real-world parameter estimates for coupled $\mathrm{GaAs}$ semiconductor waveguide resonators were given.

In addition, we have augmented Eq. (1) with a term describing a possible intersite Kerr nonlinear refractive index with a strength $Q$, of the form

$$
\begin{aligned}
f_{i s}\left(\left\{\psi_{n}\right\}\right)= & 2 \psi_{n}\left(\left|\psi_{n+1}\right|^{2}+\left|\psi_{n-1}\right|^{2}\right)+\psi_{n}^{*}\left(\psi_{n+1}^{2}+\psi_{n-1}^{2}\right) \\
& +2\left|\psi_{n}\right|^{2}\left(\psi_{n+1}+\psi_{n-1}\right)+\psi_{n}^{2}\left(\psi_{n+1}^{*}+\psi_{n-1}^{*}\right) \\
& +\left|\psi_{n+1}\right|^{2} \psi_{n+1}+\left|\psi_{n-1}\right|^{2} \psi_{n-1} .
\end{aligned}
$$

The form of this term follows from coupled-mode analysis of a waveguide array embedded in a nonlinear Kerr medium [26]; 
for simplicity we consider here a special case of the twoparameter form derived in Ref. [26] by equating the parameters to the single parameter $Q$. As shown in Ref. [27], the conservative version of Eq. (1) $\left(f_{d} \equiv 0\right)$ also appears as a rotating-wave-type approximation of a chain of anharmonic oscillators coupled with anharmonic intersite potentials, with $Q=0$ corresponding to pure onsite (Klein-Gordon chain) and $Q=1 / 2$ to pure intersite (Fermi-Pasta-Ulam chain) anharmonicity. The reason for incorporating $f_{i s}$ into our model is that this type of intersite nonlinearity may drastically reduce the Peierls-Nabarro potential for the conservative model (in the two-parameter model it may even be strictly zero at special parameter values [26]) and therefore highly facilitate the movement of even strongly localized solutions [26,27]. As we see later, the term $f_{i s}$ is not necessary for finding strongly localized moving solutions as dynamical attractors to Eq. (1) (and indeed we put $Q=0$ for the main part of this paper); however, a nonzero $Q$ increases considerably their existence region and also allows for a richer dynamical scenario.

In the conservative case $f_{d} \equiv 0$, the two conserved quantities of Eq. (1) are the total power (norm),

$$
P=\sum_{n}\left|\psi_{n}\right|^{2}
$$

and the total energy (Hamiltonian) [26], which for the general case with nonzero $V_{n}$ and $Q$ can be expressed most conveniently in the form [27]

$$
\begin{aligned}
H= & \sum_{n}\left(2 C \sqrt{A_{n} A_{n+1}} \cos \left(\phi_{n}-\phi_{n+1}\right)+\frac{A_{n}^{2}}{2}\right. \\
& +Q \sqrt{A_{n} A_{n+1}}\left\{\sqrt{A_{n} A_{n+1}}\left[2+\cos 2\left(\phi_{n}-\phi_{n+1}\right)\right]\right. \\
& \left.\left.+2\left(A_{n}+A_{n+1}\right) \cos \left(\phi_{n}-\phi_{n+1}\right)\right\}+V_{n} A_{n}\right),
\end{aligned}
$$

where we have expressed the complex mode amplitudes in terms of real action-angle coordinates, $\psi_{n}=\sqrt{A_{n}} e^{i \phi_{n}}$. In the "standard" definition of a Peierls-Nabarro (PN) barrier [28,29], one imagines a localized mode sliding with small velocity across the lattice, and the PN barrier is then defined as the difference in $H$, at fixed $P$, between the two stationary modes centered at, respectively in-between, lattice sites. In typical situations, one of these modes is stable and the other unstable, and therefore they usually correspond to $\min$ and $\max$, respectively, of an imagined PN potential. However, in some models (and notably the model (1) with nonzero $Q$ [26]), these two modes exchange stability through symmetry-breaking bifurcations under parameter variation, and there exists a small parameter regime of existence of additional, symmetrybroken, stationary solutions [26,30] which also must be taken into account when determining the "true" PN barrier.

As explained in Ref. [20], a necessary (but by no means sufficient) condition to have nontrivial localized solutions for the fully dissipative model (1) can be obtained as a restriction of the gain parameter to an interval,

$$
\delta+\frac{a-\delta+2 \sqrt{a \delta(b-1)}}{b} \leqslant g \leqslant \delta+a .
$$

The upper limit follows from the condition of existence of a stable zero-amplitude tail, and the lower limit from the condition of existence of a solution with nonzero amplitude for which the dissipative function $f_{d}\left(\left|\psi_{n}\right|^{2}\right)$ in Eq. (2) is not always negative. Notice also that the condition to have a nonvanishing interval in relation (6) imposes an additional restriction on the damping parameters $\delta, a, b$, which can be expressed as

$$
\delta \leqslant a(b-1) .
$$

Let us finally in this section also remark that expanding the dissipative function in Eq. (2) for small $x$ yields (in a cubicquintic approximation for $x=\left|\psi_{n}\right|^{2}$ such as in Refs. [22,23])

$$
f_{d}(x) \simeq g-\delta-a+(g-a b) x+\left(g-a b^{2}\right) x^{2} .
$$

However, as we will see, the relevant solutions describing moving localized modes are typically found with peak values of the order of unity or larger, and thus result essentially from the strong saturabilities of the gain and damping parts of the dissipative function on different intensity scales, and cannot be described by the cubic-quintic approximation (8).

\section{NUMERICAL RESULTS}

In order to systemize and get out the most essential features of the moving localized solutions found in certain regimes of parameter space, we from now on fix the damping parameters in Eq. (2) to have the same values as those used for the study of stationary localized modes in Ref. [20] (see also Refs. [19,31] and references therein), namely

$$
\delta=1, \quad a=2, \quad b=10 .
$$

Numerically, the largest possible existence regime (6) then becomes $1.94853<g<3$. Notably, the large value of $b$ implies that the absorption saturates at a much lower intensity than the gain. We have checked that these parameter values are not exceptional, e.g., by varying $\delta$ up to $1.5, a$ down to 0.5 , and $b$ up to 20 . Although evidently quantitative features change, the qualitative picture remains similar as long as the possible existence regime determined by relations (6) and (7) remains non-negligible. Unless otherwise noted we assume $V_{n} \equiv 0$. We first (Sec. III A) describe the scenario observed for pure onsite nonlinearity $(Q=0)$ when varying the gain amplitude $g$ and coupling constant $C$, and then (Sec. III B) discuss additional features appearing in the presence of intersite nonlinearities $(Q \neq 0)$.

In order to "seed" a moving localized solution, an initial Gaussian pulse with an imposed phase gradient was used as a trial solution. By properly adjusting parameters, such pulses were found by direct time integration of the equations of motion to belong to the basin of attraction of exact moving localized solutions with specific velocities $v$. Once found, these solutions were used as new initial conditions in continuation versus the parameters $g, C, Q$. (In practice, since the existence regime of moving solutions is larger in the presence of intersite nonlinearities, it turned out to be easier to first find moving solutions for some nonzero $Q$, which then could be continued back to the, possibly more physically interesting, case $Q=0$.)

The velocities were determined directly as $v=\Delta n_{\max } / \Delta t$, with $\Delta n_{\text {max }}$ being the translation of the soliton peak during a (large) time $\Delta t$, disregarding the initial transient before reaching the attracting state. As we see below, a moving soliton generally exhibits an internal oscillation frequency 
of its intensity $A_{n} \equiv\left|\psi_{n}\right|^{2}$, yielding oscillations also in the quantities $P$ and $H$, thereby allowing the PN barrier of the corresponding conservative system to be overcome. Assuming a single internal frequency $\omega_{b}$, a moving soliton can be assumed to have a form analogous to a moving discrete breather as defined in Ref. [32] (see also Ref. [33]),

$$
A_{n}(t)=A\left(\omega_{b} t, n-v t\right),
$$

with $A(x, y)$ being a function which is $2 \pi$ periodic with respect to its first argument and localized with respect to the second. If, for some integers $p, q$, the internal frequency and the velocity are related by a commensurability relation,

$$
p 2 \pi v=q \omega_{b},
$$

the solution will return to its initial shape after a time $T=p 2 \pi / \omega_{b}$, translated $q$ sites. As we discuss below, in the case $Q=0$ with pure onsite nonlinearity, apparently the pinning potential is strong enough to always force such a commensurability (in most cases with $p=q=1$ ). On the other hand, for nonzero $Q$ the weakening of the PN potential also allows for incommensurate regimes $(p, q \rightarrow$ $\infty$ ), where the solution, although moving with a perfectly well-defined velocity $v$, never exactly returns to its initial shape (see Sec. III B).

\section{A. The case of onsite nonlinearity, $Q=0$}

Typical results for the variation of the soliton velocity with the gain parameter $g$ in this case are shown in Fig. 1. For this particular value of the coupling constant, $C=1$, moving solutions with well-defined velocities $v$ are found for $2.0542 \lesssim g \lesssim 2.1935$. Notice that this interval is only about $13 \%$ of the interval determined by the necessary condition (6). The solutions are divided into two branches: rapidly moving solutions for weaker gain and slower solutions

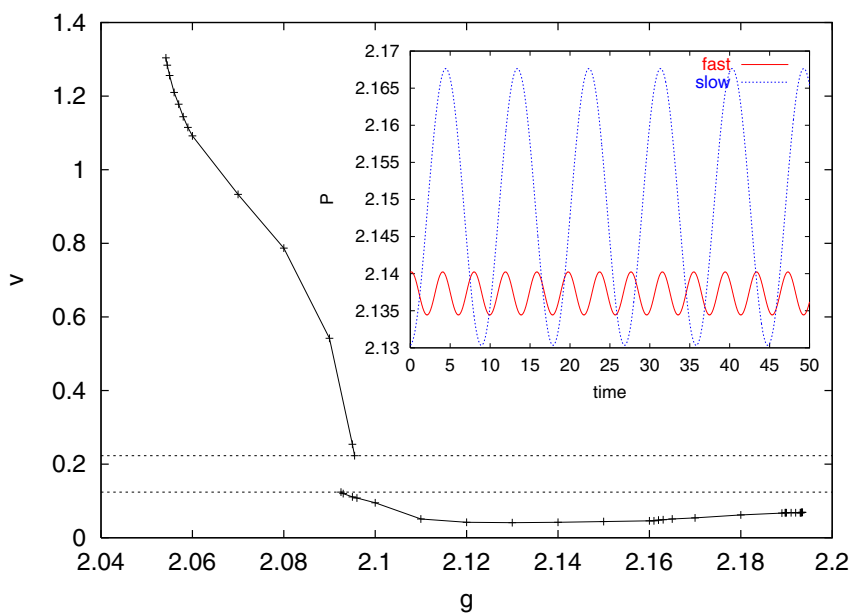

FIG. 1. (Color online) Main figure: velocity $v$ vs gain parameter $g$ for moving localized solutions of Eq. (1) with $C=1$. Other parameters are $V_{n}=Q=0$ and $f_{d}$ given by relations (2) with (9). Horizontal dashed lines indicate the velocity gap. Inset: Power (4) vs time for the bistable fast (solid [red], small oscillations) and slow (dashed [blue], large oscillations) solutions having the same $g=2.095$.

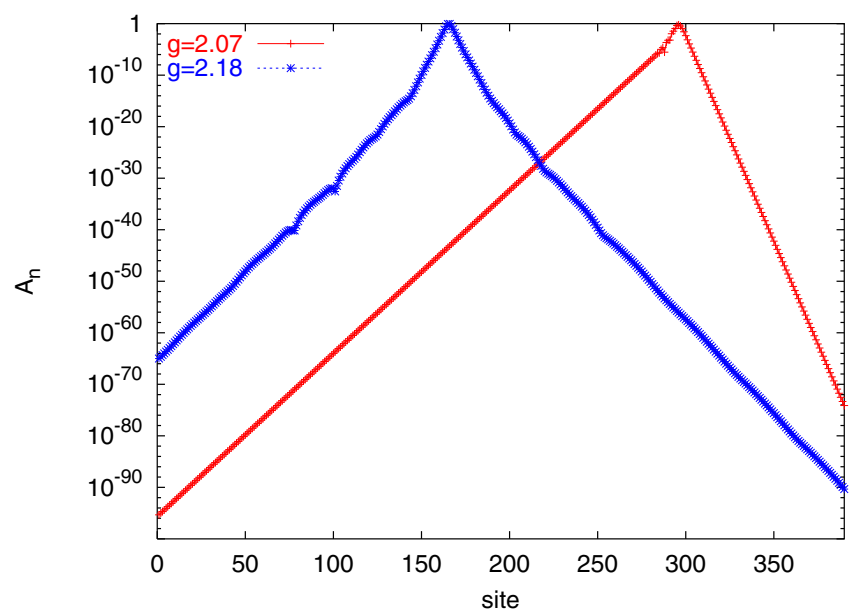

FIG. 2. (Color online) Snap shots of intensity $A_{n}$ in logarithmic scale for two right-moving solutions with $g=2.07$ (right peak [red], fast solution) and $g=2.18$ (left peak [blue], slow solution), respectively. Other parameters are the same as in Fig. 1.

for larger gain (although the velocity is not a monotonic function of $g$ for the latter branch). There is a small regime of bistability, $2.0925 \lesssim g \lesssim 2.0955$, where both solutions exist with different velocities. Notice also the "forbidden gap" of velocities in the interval $0.124 \lesssim v \lesssim 0.223$. In this aspect, the scenario is thus similar to that for the Frenkel-Kontorova model with "induced fast" and "spontaneous slow" moving breathers $[8,9]$.

Generally, as mentioned above, the moving solutions are associated with time-periodic oscillations in $P$ and $H$, and in particular the slowly moving solutions are associated with considerably larger oscillations as well as larger average values of the power $P$ (see the inset in Fig. 1 for comparison of two solutions for the same $g$ in the bistability regime).

In Fig. 2 we compare two examples of snapshots of slowly and rapidly moving solutions. Note that while both solutions are evidently exponentially localized, the tails of the rapidly moving solution show a much stronger asymmetry than those of the slow mode, with a much stronger decay in the forward than in the backward direction. This can be related to the Doppler shifts of emitted radiation as discussed in Ref. [8]. For the slower solution, the velocity is $v \approx 0.06$ so the effect is hardly noticeable, while the rapid solution moves about 15 times faster, $v \approx 0.9$. Note also the crossover from one exponential decay around the soliton center, to another, generally weaker, decay in the tails.

As seen in Fig. 1, decreasing the gain on the branch of rapid solutions leads to larger velocities, until a maximum velocity $v \approx 1.304$ is reached for $g \approx 2.0542$. With a further decrease of $g$, the solution will decay to zero after some time.

On the other hand, increasing the gain on the branch of slow solutions leads to a more complicated scenario. The power oscillation amplitude will increase, as well as its average value, and develop strongly anharmonic features in contrast to the nearly harmonic oscillations shown for smaller gain in Fig. 1. For $g \gtrsim 2.13$ the oscillations become double-peaked. While, for the case $C=1$ shown in Fig. 1, the oscillations are 1:1 locked with the lattice translations 


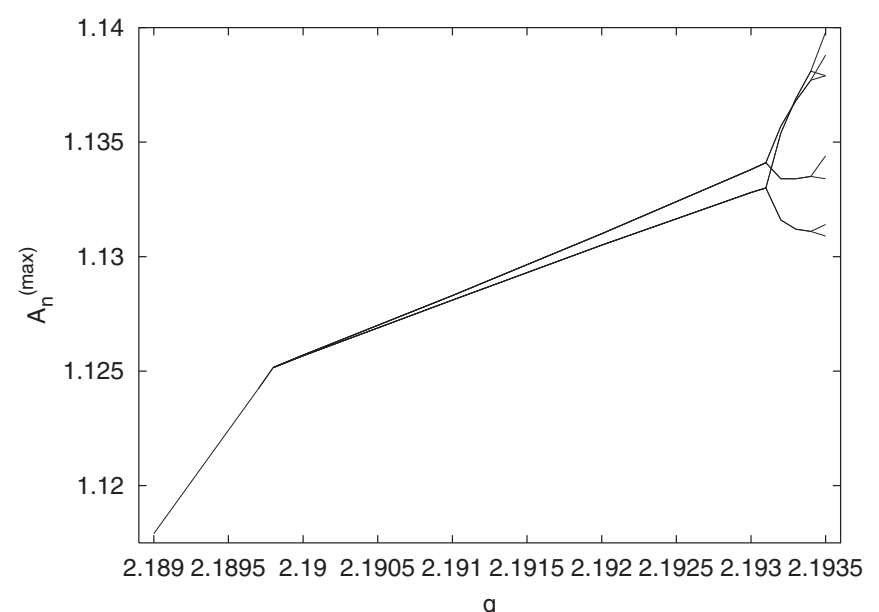

FIG. 3. Maximum intensity $A_{n}^{(\max )}$ versus gain parameter $g$ for different lattice sites, illustrating the locking between internal oscillations and lattice translations for steadily moving solutions, and the period-doubling scenario in the upper end of the existence regime. Parameter values are the same as in Fig. 1.

$[(p, q)=(1,1)$ in Eq. $(11)]$ in most of the existence regime for the moving solutions, a period-doubling scenario is observed when increasing $g$ even further. Here, we found a solution with period $2[(p, q)=(1,2)]$ in an interval $2.1898 \lesssim g \lesssim 2.1931$, period $4[(p, q)=(1,4)]$ when $2.1932 \lesssim g \lesssim 2.1934$, period $8[(p, q)=(1,8)]$ in a small interval around $g \approx 2.1935$, etc. The period-doubling scenario is illustrated in Fig. 3. For a further increase of $g$ the power oscillations become irregular, resulting in an apparently chaotically moving solution which, similar to the examples shown in Refs. [8,15], may move with almost constant velocities for some time, then get trapped for some brief interval, after which it restarts its motion in an apparently random direction. For these parameter values, the randomly walking solution appears only in a small interval of $g$, and a further increase $(g \gtrsim 2.205)$ yields a splitting of the soliton into two oppositely moving fronts ("switching waves" $[20,21])$ so that all lattice sites will end up at a constant, nonzero, intensity.

With the decrease of the coupling constant $C$, the range of $g$ where steadily moving localized solitons exist narrows, and for $C \lesssim 0.45$ we were not able to find any moving solitons at all. Moreover, all solitons generally get slower when $C$ decreases, which is consistent with the interpretation of a larger PN barrier for strongly discrete lattices. In particular, for $C=0.46$ (close to the lower existence boundary) we observe moving solitons for $2.0528 \lesssim g \lesssim 2.0602$ with velocities $0.045 \lesssim v \lesssim 0.064$ (monotonously decreasing with $g$ ). Also here there are two main branches of solution as for $C=1$, however without multistability. Instead, the "fast" branch now also exhibits period-doubling bifurcations into a regime of chaotically moving solutions starting at $g \approx 2.0564$, while the "slow" branch first appears at $g \approx 2.0600$. Interestingly, in addition to the period doublings there are also windows of higher periodic regularly moving solutions inside the regime of chaotic solutions, analogous to the standard Feigenbaum scenario. For example, we found a period-3 window $[(p, q)=(1,3)]$ for $2.0569 \lesssim g \lesssim 2.0574$, bifurcating to period $6[(p, q)=(1,6)]$
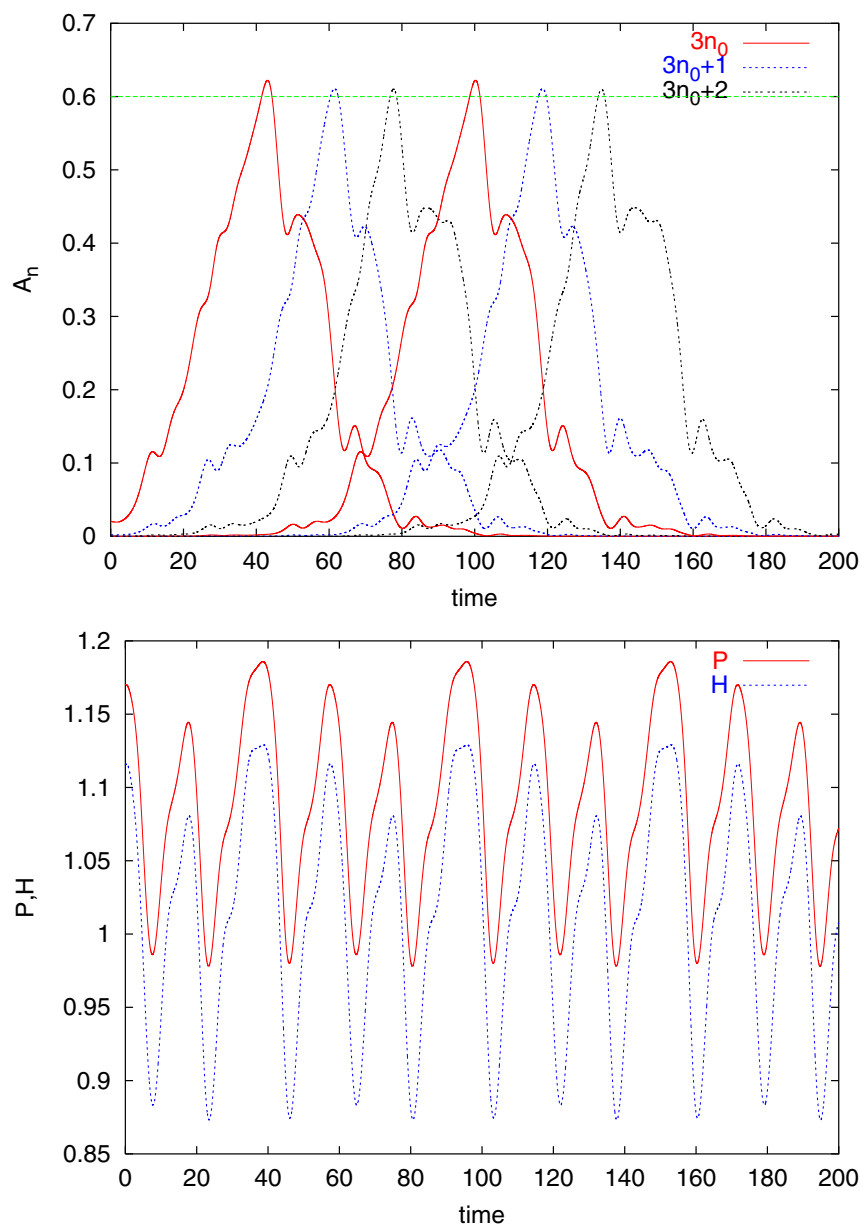

FIG. 4. (Color online) Dynamics of a moving soliton with velocity $v \approx 0.052$ in the period-3 window $[(p, q)=(1,3)]$ for $C=0.46$ and $g=2.0574$ (other parameters same as in Fig. 1). Upper figure: intensity $A_{n}$ vs time for six consecutive sites (larger $n$ corresponds to later peak time). Note the identical curve shapes for every third site (the horizontal line at 0.6 is a guide to the eye). Lower figure: the corresponding oscillations of the quantities $P$ (4) (solid [red] line) and $H(5)$ (dashed [blue] line).

around $g \approx 2.0575$, etc. The dynamics of a stable moving period-3 solution is illustrated in Fig. 4. Note that even though three different types of peaks are clearly visible in the (strongly anharmonic) oscillations of power and Hamiltonian, the resulting differences in local intensity peak heights are hardly distinguished on the scale of Fig. 4.

\section{B. The case of intersite nonlinearity, $Q \neq 0$}

As mentioned above, including a nonzero $Q$ with an intersite nonlinearity (3) for the conservative system (1) $\left(V_{n} \equiv 0\right.$, $f_{d} \equiv 0$ ) effectively reduces the PN barrier and enhances mobility of localized modes. The PN barrier is expected to be smallest in the regime where the site-centered and bondcentered solutions exchange stability; the exact location of this exchange depends slightly on the ratio $P / C$ so that the upper limit for stability of the site-centered solution is $Q_{c} \approx 0.25$ for $P / C \lesssim 1$, and $Q_{c} \approx 0.26$ for $P / C \gtrsim 10$ (cf. [26,34]). 


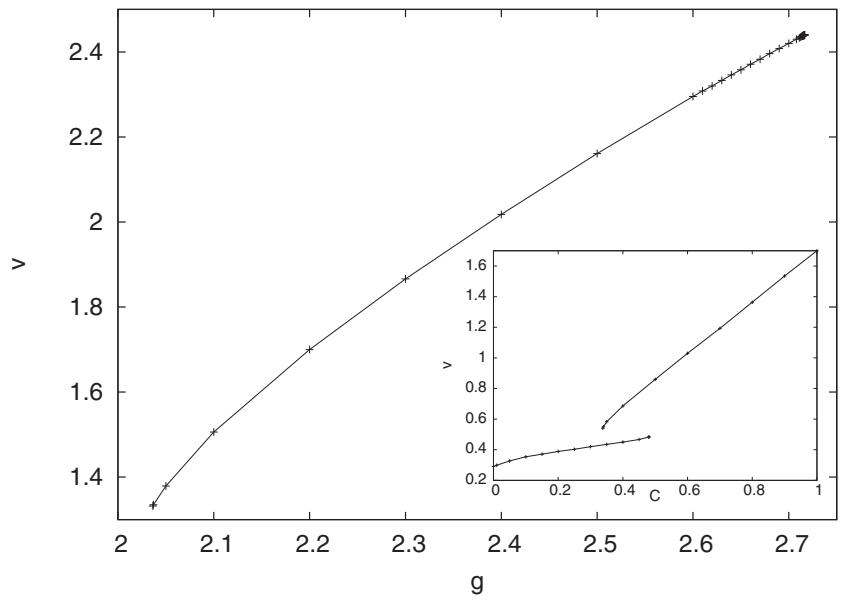

FIG. 5. Main figure: velocity $v$ vs gain parameter $g$ for moving localized solutions of Eq. (1) with $C=1, Q=0.4$, and other parameters same as in Fig. 1. Inset: velocity vs coupling constant $C$ for fixed gain parameter $g=2.2$ and other parameters the same as in the main figure.

For the dissipative system with parameter values as in relation (9), we correspondingly observe a considerable enhancement in the existence regimes for moving solitons when $Q \gtrsim 0.2$. So, for example, taking $Q=0.4$ and $C=1$ we find a continuous branch of rapidly moving solutions with small-amplitude oscillations in the full interval $2.0363 \lesssim g \lesssim$ 2.7166, with velocities monotonously increasing with $g$ from $v \approx 1.332$ to $v \approx 2.440$ (Fig. 5). Thus, compared to the case $Q=0$ of Fig. 1, the existence interval in $g$ is not only five times larger, but also the dependence $v(g)$ is the opposite (with regard to the fast branch).

Also the lower existence boundary in $C$ for moving solitons becomes considerably smaller when $Q \neq 0$. Taking again, as an example, $Q=0.4$ and fixing $g=2.2$, we find (see inset in Fig. 5) that although the branch of stable rapidly moving solutions continued from $C=1$ terminates at $C \approx 0.338$, there is another branch of slow, large-amplitude oscillation solutions appearing at $C \approx 0.481$ (i.e., again with an interval of bistability), which can be continued all the way down to $C=0$. In this limit, the solution moves with nonzero velocity $(v \approx 0.291)$ solely due to the influence of the nonlinear intersite couplings.

Another interesting effect of the weaker pinning potential is the appearance of parameter regimes where the relation (11) between internal oscillation frequency and soliton velocity becomes incommensurate. To illustrate this, we now fix $g=$ 2.2 and $C=0.3$ and continue the moving solutions vs $Q$ (from Sec. III A we know that no stable moving solutions exist for these parameter values when $Q=0$ ). A fast, stable smalloscillation solution exists for these parameter values only in the interval $0.264 \lesssim Q \lesssim 0.350$, while the slower solution with large-amplitude oscillations is found to propagate with a welldefined velocity down to $Q \approx 0.2095$. The oscillations and the velocity are $1: 1$ locked $[(p, q)=(1,1)]$ for $Q \gtrsim 0.265$, where a Hopf-type bifurcation appears and the oscillations develop an additional modulation frequency, incommensurate with the translational dynamics as illustrated in Fig. 6.
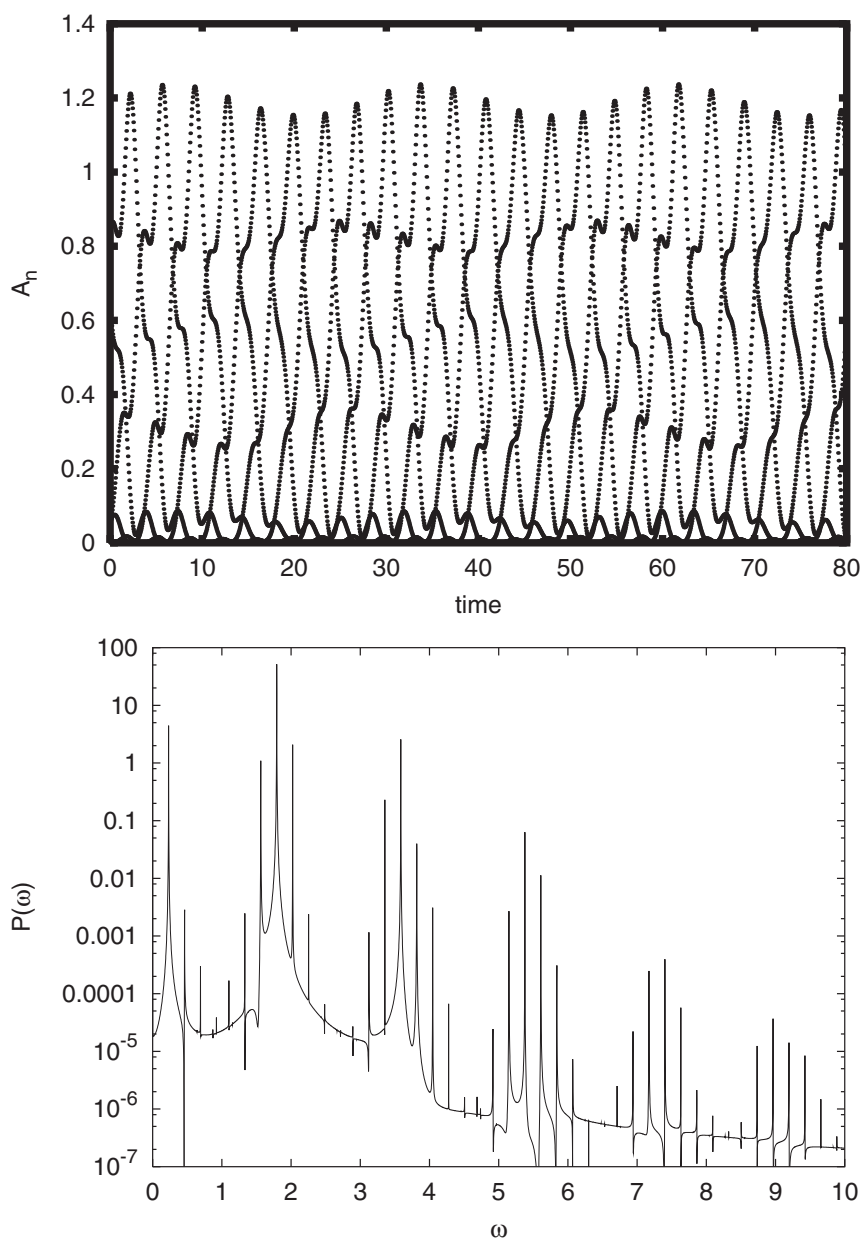

FIG. 6. Dynamics of a moving "slow" soliton for $Q=0.264$, $C=0.3$, and $g=2.2$ (other parameters same as in Fig. 1). Upper figure: intensity $A_{n}$ vs time for 23 consecutive sites (larger $n$ corresponds to later peak time). Notice that while peaks are equidistant, corresponding to a well-defined velocity $v \approx 0.285$, the modulation of the peaks is apparently incommensurate; i.e., no two peaks corresponding to different sites have identical shape. Lower figure: Fourier spectrum of the corresponding oscillations of the power $P$ (4), calculated over 40000 time units. The main peak at $\omega=2 \pi v \approx 1.793$ corresponds to the translational motion, while the first peak at $\omega \approx 0.231$ corresponds to the modulation. All other peaks seen are linear combinations of these.

The quasiperiodically moving slow soliton can be found for $0.224 \lesssim Q \lesssim 0.264$ (note that this corresponds roughly to the regime where the PN potential of the corresponding conservative system is expected to be weakest as discussed above). However, as the modulation frequency varies (generally increases as $Q$ decreases in this interval), we also find small intervals of nontrivially phase locked states, where the two fundamental frequencies lock to rational values (i.e., analogous to Arnol'd tongues). As an example, we show in Fig. 7 the 2:5-locked state $[(p, q)=(2,5)]$ which is a stable attractor for $0.2281 \lesssim Q \lesssim 0.2285$ with other parameters as above.

Decreasing $Q$ further leads to a larger interval of $(p, q)=$ $(1,2)$ locking, $0.214 \lesssim Q \lesssim 0.227$, which for a further decrease undergoes a sequence of period-doubling bifurcations 

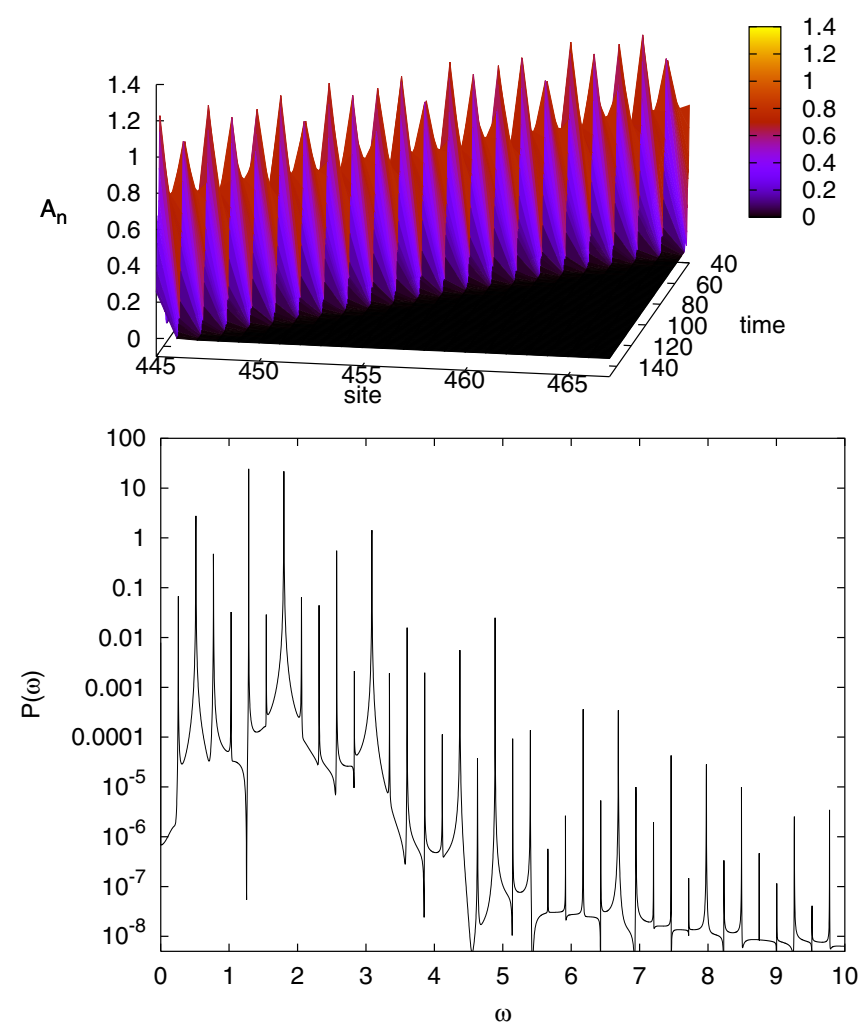

FIG. 7. (Color online) Dynamics of a soliton moving with velocity $v \approx 0.205$ in the $(p, q)=(2,5)$ phase-locked regime for $Q=0.2285$ (other parameters same as in Fig. 6). Upper figure: intensity $A_{n}$ vs time. Note that the pattern periodically repeats itself after translating $q=5$ sites. Lower figure: Fourier spectrum of the oscillations of the power $P$ (4), calculated over 40000 time units. The peak at $\omega_{1}=2 \pi v \approx 1.286$ corresponds to the translational motion, while the second peak at $\omega_{2} \approx 0.514$ corresponds to the main modulation. The first peak is $\omega_{1}-2 \omega_{2}=\omega_{2} / 2$, and all other peaks seen are multiples of this frequency.

leading to a chaotically moving solution for $Q \lesssim 0.209$, similarly as described above for $Q=0$. Finally, at $Q \approx 0.15$ it gets trapped into a mode oscillating around a fixed site, similar to that described in Refs. $[8,15]$.

\section{Effects of disorder}

Finally, we illustrate that the existence of moving lattice solitons is not crucially dependent on having a perfect lattice, but they also survive in the presence of weak disorder. Generally, disordered photonic structures are of large current interest, e.g., in the contexts of random lasers and Anderson localization of light; see, e.g., recent reviews [35,36]. Here, we choose the onsite potential $V_{n}$ in Eq. (1) to have a uniform distribution in the interval $\left[-V_{0}, V_{0}\right]$, and launch initially a soliton found as an attractor in the regular lattice. If the disorder is sufficiently weak, the soliton settles into a new attracting moving mode after a short transient, as illustrated in Fig. 8. For the parameter values chosen in Fig. 8, which for $V_{0}=0$ belong to the branch of fast solitons for $Q=0$ in Fig. 1, a moving attracting state appears for $V_{0} \lesssim 0.1$, while the soliton generally gets trapped for a slightly stronger disorder. The velocity of the soliton in the disordered lattice in Fig. 8 is only
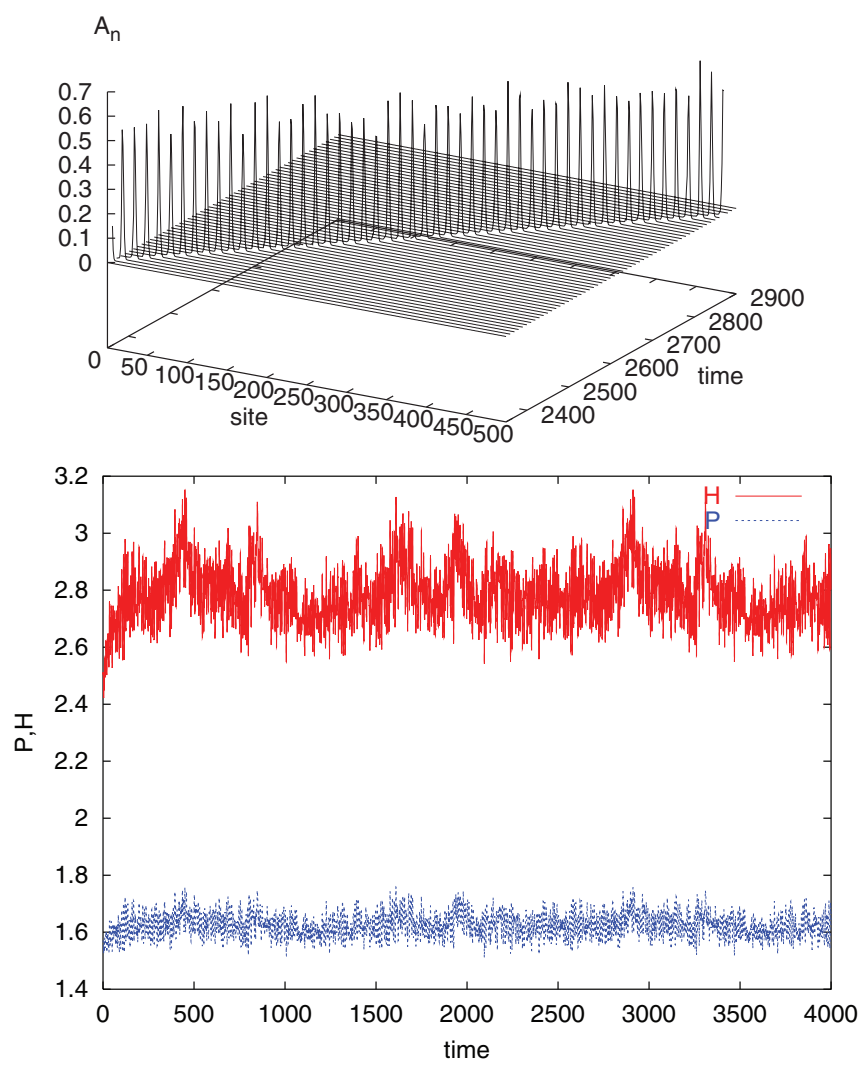

FIG. 8. (Color online) Dynamics of a soliton moving with velocity $v \approx 0.977$ in a lattice with uniform disorder of strength $V_{0}=0.1$, for $g=2.06$ and other parameters same as in Fig. 1. The soliton appears as an attractor using the soliton for $V_{0}=0$ as initial condition. Upper figure: intensity $A_{n}$ vs time. Only a part of the dynamics is shown for a lattice with 2405 sites and periodic boundary conditions. Lower figure: the corresponding oscillations of the quantities $P$ (4) (lower [blue] curve) and $H$ (5) (upper [red] curve). Note that after an initial transient, the patterns periodically repeat themselves after 2462 time units, corresponding to one round trip in the lattice.

slightly smaller than for the corresponding parameter values in the regular lattice $(v \approx 0.977$ compared to $v \approx 1.092$ ). From the lower part of Fig. 8, we see apparently irregular oscillations of power and Hamiltonian, compensating for the irregularities in the disordered lattice so that the moving soliton still can travel with a constant velocity. Note from Fig. 8 that the oscillation patterns exactly repeat themselves after one round trip in the periodic lattice. Thus, even in a disordered lattice the soliton may at each point adjust its internal parameters according to its local environment, in order to travel with constant velocity.

As could be expected, the critical disorder below which a traveling mode can be sustained is generally considerably smaller for the slow, large-amplitude oscillation modes. For example, with the parameter values of Fig. 1, we found that the moving solution with $g=2.18$ in Fig. 2 keeps traveling with constant velocity for many round trips in the lattice when $V_{0}=$ 0.0006 , but gets trapped for slightly larger disorder. Also in this case the velocity gets slightly smaller in presence of disorder ( $v \approx 0.059$ compared to $v \approx 0.062$ for the above parameter values). 


\section{CONCLUSIONS}

In conclusion, we showed that exact traveling, exponentially localized, discrete solitons exist as stable dynamical attractors in a model for active waveguide arrays with Kerr nonlinearity, supported only by the intrinsic gain and damping, without explicit external forcing or symmetry-breaking perturbations. The traveling soliton self-adjusts its internal parameters during the motion in order to travel with a constant velocity, leading to oscillations of its total power and Hamiltonian. In most cases these oscillations are 1:1 locked to the translational motion, but in certain regimes period-doubling bifurcations to chaotically walking modes are observed, and likewise Hopf bifurcations to incommensurate oscillations and nontrivial phase-locking intervals may appear if also intersite Kerr nonlinearities are present. For pure onsite nonlinearity traveling solitons exist only in a rather narrow parameter regime, which widens considerably with the inclusion of intersite nonlinearities, thereby reducing the Peierls-Nabarro potential obstructing the motion in the corresponding conservative system. The moving solitons can be divided into two types, between which bistability appears in certain regimes: fast modes with small oscillations and slow modes with large oscillations. The former may even survive as exact traveling modes if a reasonably strong disorder is included, while the latter are highly sensitive to trapping.

In our model, the saturable gain and absorption result from the properties of the active optical fibers as described in Ref. [21]. It is clear that, in this model, all parameter values describing the gain and damping need to be nonzero for stable traveling localized solitons to exist; e.g., they would not exist for purely linear damping or purely linear gain. Evidently, it would be highly interesting to understand more precisely the necessary conditions for existence of traveling intrinsic gain-driven localized modes in more general physical lattice systems, not necessarily restricted to optics. One particularly interesting example concerns the so-called quodons in layered crystals such as mica, where it has been suggested that nonlinear lattice excitations could travel for macroscopic distances by an energy gain resulting from the lattice being in a metastable configuration [37]. In the optical context, extensions to nonKerr nonlinearities and higher dimensions are also relevant, particularly as it is known that saturable [38] or quadratic [39] nonlinearities may considerably decrease the Peierls-Nabarro barriers and increase mobility of localized modes also for two-dimensional lattices in the conservative case.

\section{ACKNOWLEDGMENTS}

The authors thank A. V. Yulin and S. K. Turitsyn for useful discussions. M.J. also thanks M. Marklund for some earlier ideas in this direction. M.J. thanks the School of Engineering and Applied Science, Aston University, for its kind hospitality and acknowledges support from the Swedish Research Council. J.E.P. appreciates the support of the ERC project ULTRALASER. S.D. was supported by Marie Curie Fellowship project INDIGO.
[1] N. Akhmediev and A. Ankiewicz, eds., Dissipative Solitons, Lecture Notes in Physics Vol. 661 (Springer, Berlin, 2005).

[2] N. Akhmediev and A. Ankiewicz, eds., Dissipative Solitons: From Optics to Biology and Medicine, Lecture Notes in Physics Vol. 751 (Springer, Berlin, 2008).

[3] T. Ackemann, W. J. Firth, and G.-L. Oppo, in Advances in Atomic Molecular and Optical Physics, edited by E. Arimondo, P. R. Berman, and C. C. Lin, Vol. 57, (Academic Press, London, 2009), p. 323.

[4] S. Flach and A. V. Gorbach, in Ref. [2], p. 289.

[5] N. N. Akhmediev and A. Ankiewicz, Solitons: Nonlinear Pulses and Beams (Chapman \& Hall, London, 1997).

[6] S. Flach and A. V. Gorbach, Phys. Rep. 467, 1 (2008).

[7] D. E. Pelinovsky, Localization in Periodic Potentials: From Schrödinger Operators to the Gross-Pitaevskii Equation, London Mathematical Society Lecture Note Series Vol. 390 (Cambridge University Press, Cambridge, 2011).

[8] J. L. Marín, F. Falo, P. J. Martínez, and L. M. Floría, Phys. Rev. E 63, 066603 (2001).

[9] P. J. Martínez, M. Meister, L. M. Floría, and F. Falo, Chaos 13, 610 (2003).

[10] M. Meister and L. M. Floría, Eur. Phys. J. B 37, 213 (2004).

[11] D. Zueco, P. J. Martínez, L. M. Floría, and F. Falo, Phys. Rev. E 71, 036613 (2005).

[12] U. Peschel, O. Egorov, and F. Lederer, Opt. Lett. 29, 1909 (2004).
[13] O. A. Egorov, F. Lederer, and Yu. S. Kivshar, Opt. Express 15, 4149 (2007).

[14] A. Yulin and A. Champneys, Disc. Cont. Dyn. Syst., Ser. S 4, 1341 (2011).

[15] O. A. Egorov and F. Lederer, Opt. Lett. 38, 1010 (2013).

[16] L. Q. English, R. B. Thakur, and R. Stearrett, Phys. Rev. E 77, 066601 (2008).

[17] L. Q. English, F. Palmero, A. J. Sievers, P. G. Kevrekidis, and D. H. Barnak, Phys. Rev. E 81, 046605 (2010).

[18] L. Q. English, F. Palmero, J. F. Stormes, J. Cuevas, R. CarreteroGonzález, and P. G. Kevrekidis, Phys. Rev. E 88, 022912 (2013).

[19] N. V. Vysotina, N. N. Rosanov, V. E. Semenov, S. V. Feodorov, and A. N. Shatsev, Opt. Spectrosc. 105, 436 (2008).

[20] Al. S. Kiselev, An. S. Kiselev, and N. N. Rozanov, Opt. Spectrosc. 105, 547 (2008).

[21] N. N. Rosanov, Spatial Hysteresis and Optical Patterns (Springer, Berlin, 2002).

[22] N. K. Efremidis and D. N. Christodoulides, Phys. Rev. E 67, 026606 (2003)

[23] N. K. Efremidis and D. N. Christodoulides, in Ref. [1], p. 309.

[24] Y. Kominis, P. Papagiannis, and S. Droulias, Opt. Express 20, 18165 (2012).

[25] J. E. Prilepsky, A. V. Yulin, M. Johansson, and S. A. Derevyanko, Opt. Lett. 37, 4600 (2012).

[26] M. Öster, M. Johansson, and A. Eriksson, Phys. Rev. E 67, 056606 (2003). 
[27] M. Johansson, Physica D 216, 62 (2006).

[28] J. C. Eilbeck, in Computer Analysis for Life Science: Progress and Challenges in Biological and Synthetic Polymer Research, edited by C. Kawabata and A. R. Bishop (Ohmsha, Tokyo, 1986), p. 12.

[29] Yu. S. Kivshar and D. K. Campbell, Phys. Rev. E 48, 3077 (1993).

[30] S. Aubry, Physica D 216, 1 (2006).

[31] N. N. Rosanov, in Ref. [1], p. 101.

[32] S. Flach and K. Kladko, Physica D 127, 61 (1999).

[33] S. Aubry and T. Cretegny, Physica D 119, 34 (1998).

[34] P. Jason and M. Johansson, Phys. Rev. A 88, 033605 (2013).

[35] D. S. Wiersma, Nat. Photon. 7, 188 (2013).
[36] M. Segev, Y. Silberberg, and D. N. Christodoulides, Nat. Photon. 7, 197 (2013).

[37] F. M. Russell, in Localized Excitations in Nonlinear Complex Systems: Current State of the Art and Future Perspectives, edited by R. Carretero-González, J. Cuevas-Maraver, D. Frantzeskakis, N. Karachalios, P. Kevrekidis, and F. PalmeroAcebedo (Springer, Cham, 2014), p. 289.

[38] R. A. Vicencio and M. Johansson, Phys. Rev. E 73, 046602 (2006); U. Naether, R. A. Vicencio, and M. Johansson, ibid. 83, 036601 (2011)

[39] H. Susanto, P. G. Kevrekidis, R. Carretero-González, B. A. Malomed, and D. J. Frantzeskakis, Phys. Rev. Lett. 99, 214103 (2007). 